\title{
EDITORIAL
}

\section{Why prevent Invasive Candida Infections?}

\section{Journal of Perinatology (2008) 28, 385-388; doi:10.1038/jp.2008.38}

In the Journal, Weitkamp et al. describe their approach to the application of fluconazole prophylaxis to high-risk preterm infants to prevent invasive Candida infections (ICIs). This group examined their incidence of ICI, and instituted prophylaxis at a cutoff for ICI based on gestational age and birth weight, and reported a significant decrease in ICI, elimination of Candidarelated mortality and a reduction in empiric antifungal use. This paper gives insight into the application of this evidence-based therapy to neonatal intensive care units (NICUs).

\section{Why prevent ICI?}

There are nearly 30000 preterm infants $<1000 \mathrm{~g}$ and/or $\leqslant 27$ weeks gestation born each year in the United States (National Vital Statistics, Center of Disease Control and Prevention-CDC, 2004), translating into approximately 2000 to 3000 ICI, 200 to 400 Candida-related deaths and 900 to 1200 infants developing neurodevelopmental impairment (NDI) associated with ICI. ${ }^{1,2}$

Prevention of ICI is critical as NDI or death occurs in $73 \%$ of infected infants $<1000 \mathrm{~g} .{ }^{3} \mathrm{NDI}$ occurred in $57 \%$ of survivors with bloodstream or cerebrospinal fluid (CSF) ICI and Candida bloodstream infections have the highest associated NDI compared to other infections (Figure 1). ${ }^{4}$ In this analysis, prompt or empiric therapy did not decrease NDI in these patients.

In infants $<1000 \mathrm{~g}$ with ICI, mortality rates range from 26 to $66 \%$ of the control patients in the prophylaxis studies. ${ }^{5-13}$ There is a marked difference in mortality between infants $<1000 \mathrm{~g}$ and larger infants. A recent analysis using ICD-9 codes reported a crude mortality rate of $26 \%$ with ICI compared to $13 \%$ for other infants $<1000 \mathrm{~g}$, and for infants $>1000 \mathrm{~g}$ with ICI, mortality was $2 \%$ compared to $0.4 \%$ among infants without candidiasis. ${ }^{1,14,15}$

In additional to the morbidity and mortality, two recent casecontrolled studies have examined the effect of ICI on cost of hospitalization and length of hospital stay. ${ }^{14,15}$ They are limited in being based on ICD-9 codes which often may not be coded for all ICI. The mean increase in hospital costs was $\$ 39045$ for infants $<1000 \mathrm{~g}$ with no difference in length of stay, and for infants $\geqslant 1000 \mathrm{~g} \$ 122302$ with an additional length of stay of 16 days. ${ }^{15}$

\section{Prophylaxis}

The efficacy and safety of fluconazole prophylaxis in preterm infants has been reported in over 2400 patients from four randomized controlled trials ${ }^{5-8}$ and five retrospective studies ${ }^{9-13}$ without any significant adverse effects or emergence of resistance. Meta-analysis of these studies demonstrates that fluconazole prophylaxis reduced the risk of developing ICI in high-risk infants $<1000$ g by $91 \%$ (odds ratio (OR), 0.09; $95 \%$ confidence interval (CI), 0.04 to $0.24 ; P=0.0004$ ) and all infants $<1500 \mathrm{~g}$ by $85 \%$ (OR, 0.15; $95 \%$ CI, 0.08 to $0.26 ; P<0.0001$ ). Candida-related mortality was decreased by $96 \%$ (OR, 0.04; $95 \%$ CI, 0.01, 0.31; $P=0.0055)$ and overall mortality rate by $25 \%(11 \%$ in the fluconazole-treated infants compared with $16.3 \%$ in the control patients) (OR, $0.75 ; 95 \% \mathrm{CI}, 0.58$ to $0.97 ; P=0.029)$. Healy et al. ${ }^{9}$ also reported the elimination of Candida-related mortality in any patient in their NICU when fluconazole prophylaxis was targeted to infants $<1000 \mathrm{~g}$.

Fluconazole prophylaxis is extremely cost effective. Uko et al. ${ }^{12}$ examined the cost with fluconazole prophylaxis and showed a significant cost benefit of $\$ 516702$ over 18 months in their NICU. At our institution, pharmacy costs of one dose are \$18 (M Buck, PharmD, personal communication), making the cost of the average time of prophylaxis of 4 to 6 weeks ( 8 to 12 doses) between $\$ 144$ and 216 per patient.

\section{Resistance and safety}

Some of the issues related to prophylaxis include side effects and resistance. In bone marrow transplant patients, fluconazole prophylaxis has decreased both ICI and mortality while fungal resistance remains low around 5\%. ${ }^{16}$ Neonatal prophylaxis studies have not reported a significant change or emergence of resistant species over the course of prophylaxis, during the study periods of 2 to 3 years, or over a 5-year period encompassing two studies. ${ }^{5,7}$ Furthermore, there was no emergence or increase in the incidence of colonization or infection due to Candida glabrata or Candida krusei reported in any studies as well as a recent single center analysis of 10 years ( 4 years prior to and 6 years post-fluconazole prophylaxis). ${ }^{17}$

Fluconazole prophylaxis at higher doses $\geqslant 6 \mathrm{mg} \mathrm{kg}^{-1}$ and frequency may be associated with the development of $C$. parapsilosis resistance. ${ }^{18,19}$ This finding suggests that it may be important to focus fluconazole prophylaxis use in only select high-risk NICU patients and primarily for prophylaxis and a different antifungal for treatment or empiric therapy, such as amphotericin B when able, as this will limit overall fungal exposure to fluconazole and possibly prevent the emergence of resistance. 
This study did not demonstrate any side effects, including the incidence of cholestasis, which is consistent with results from the randomized controlled studies. One of the retrospective studies reported a higher incidence of cholestasis in the fluconazole prophylaxis patients that was transient with no difference at discharge while another retrospective study demonstrated a lower incidence of cholestasis. ${ }^{12,13}$ Since there were no significant differences in direct bilirubin or liver enzymes in the four randomized placebo-controlled trials, it may be that in the one retrospective study other factors present during the study period increased the likelihood of cholestasis.

\section{What is the incidence and severity of ICI in your NICU?}

Most studies only report bloodstream infections, and fail to account for meningitis, urinary tract infections (of which one-third have renal abscess involvement) and sterile body fluids such as peritoneal infections complicating NEC and focal bowel perforations. Furthermore, some cases are missed as meningitis is likely underreported due to lack of CSF data at the time of sepsis

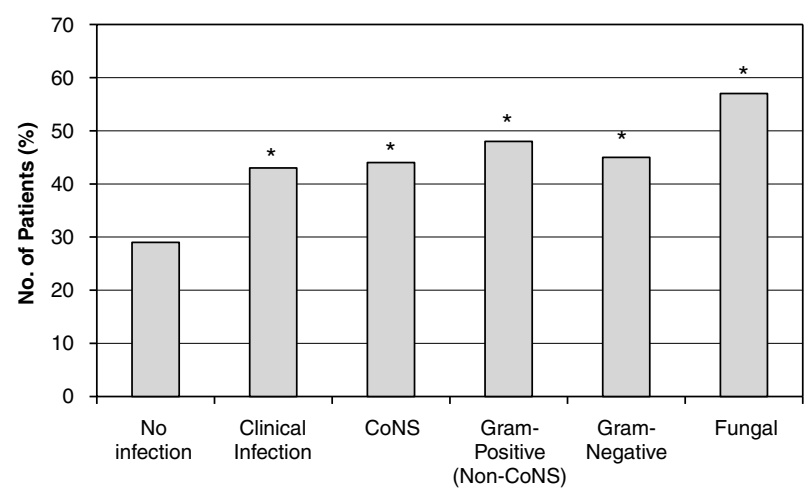

Figure 1 Neurodevelopmental impairment and bloodstream infection in infants $<1000$ g. ${ }^{4} * P \leqslant 0.001$ compared to no infection group. evaluations, some cases are not detected until autopsy (which often does not occur) and Candida pneumonia is difficult to definitively diagnose. $^{20}$

This paper demonstrates the importance of examining ICI incidence in each NICU as many flaws exist when solely relying on the literature for incidence. This NICU reported that their rate of Candida bloodstream infections was $6.8 \%$, while the rate of all ICI was $10 \%$ for infants $<1000 \mathrm{~g}$. In the largest analysis from 132 NICUs, the median rate of Candida bloodstream infections was $7.5 \%$ in infants $<1000 \mathrm{~g}$, and similar to this study results, the incidence of all ICI would be approximately $4 \%$ higher when including meningitis and urine tract infections. ${ }^{1,21}$

Gestational age has a more linear relationship to ICI compared to birth weight and captures the highest-risk patients. ${ }^{22-24}$ For example, examining growth charts, a 24-week gestation infant could be between 468 to $940 \mathrm{~g}$ (3rd to 97 th percentiles). ${ }^{25}$ As this study demonstrated, by examining the incidence of ICI by each gestational age and birth weight, they were able to see where the rates in their population fell to zero. Table 1 illustrates an infectious control approach for each NICU to analyze their incidence of ICI with infection-related mortality and NDI.

\section{Who should receive antifungal prophylaxis?}

The question many have raised is, who would benefit from receiving antifungal prophylaxis? Several factors should go into that decision including incidence, mortality and NDI.

(1) Targeted prophylaxis should be given to all infants $<1000 \mathrm{~g}$ and/or $\leqslant 27$ weeks while they require intravenous (IV) access (peripheral or central) starting on day 1 up to 6 weeks of life. This subpopulation of preterm infants has high mortality and NDI, and this approach has demonstrated efficacy and safety without the emergence of resistance in randomized controlled trials, while eliminating Candida-related mortality.

Table 1 Invasive Candida infection (ICI ) surveillance chart

\begin{tabular}{|c|c|c|c|c|c|c|c|}
\hline Gestational age & All ICI (\%) & Mortality & $N D I$ & Bloodstream infections (\%) & UTI (\%) & Meningitis (\%) & Other $*(\%)$ \\
\hline \multicolumn{8}{|l|}{22} \\
\hline \multicolumn{8}{|l|}{23} \\
\hline \multicolumn{8}{|l|}{24} \\
\hline \multicolumn{8}{|l|}{25} \\
\hline \multicolumn{8}{|l|}{26} \\
\hline \multicolumn{8}{|l|}{27} \\
\hline \multicolumn{8}{|l|}{28} \\
\hline \multicolumn{8}{|l|}{29} \\
\hline 30 & & & & & & & \\
\hline
\end{tabular}

Abbreviations: ICI, invasive Candida infections; NDI, neurodevelopmental impairment; UTI, urinary tract infections.

NDI (one or more of the following: PDI or $\mathrm{MDI}<70$, cerebral palsy, blindness and deafness).

*0ther infections (peritoneal and/or other sterile body fluid). 
(2) Even in a NICU with overall low rates of ICI $(<2 \%)$, infants $\leqslant 26$ weeks are likely high-risk and would benefit from prophylaxis. Incidence and outcomes by gestational age should be examined and tracked (Table 1). ICI can be analyzed by filling out Table 1 at institutions with low rates to determine the gestational age range in which ICI does occur and to identify those infants who should receive prophylaxis. There is likely a gestational age cutoff wherein ICI falls to zero. If NICUs do not have neurodevelopmental outcome data, prophylactic treatment of high-risk infants $<1000 \mathrm{~g}$ or $\leqslant 27$ weeks should strongly be considered as treatment of documented infections does not always prevent the NDI and mortality of these infections. ${ }^{3,4}$

(3) NICUs with high rates in infants 1000 to $1500 \mathrm{~g}$ may choose prophylaxis in these infants. A targeted approach to infants with a central venous catheter (CVC) or on antibiotics for $>3$ days has been used in retrospective studies. ${ }^{10,12}$

\section{Dosage and schedule}

The dosage used in this study was $3 \mathrm{mg} \mathrm{kg}^{-1}$ intravenously twice a week until IV access (peripheral or central) was no longer needed. This study safely extended prophylaxis beyond 6 weeks continuing up to 9 weeks in those infants who required IV access longer. Manzoni et al. ${ }^{8}$ in their multicenter randomized clinical trials (RCT) demonstrated that 3 or $6 \mathrm{mg} \mathrm{kg}^{-1}$ are equally effective. However, dosing with $3 \mathrm{mg} \mathrm{kg}^{-1}$ is preferable for the following two reasons: (1) drug concentrations in the skin, lung and mucous membranes are greater than plasma levels (therefore larger doses may be unnecessary), (2) the use of higher doses may foster development fungal resistance. Furthermore, the goal of prophylaxis is to use the lowest effective dose (usually $50 \%$ of treatment dose). In a recently published RCT, twice weekly dosing was as effective in preventing infection as more frequent dosing. ${ }^{7}$ Therefore, $3 \mathrm{mg} \mathrm{kg}^{-1}$ given twice a week is the optimal dosing schedule, maximizing efficacy, safety and cost.

The computerized system order entry system designed to not miss any potential patients was used in this study for quality improvement. In addition, we administer fluconazole prophylaxis twice weekly on the same days, every Tuesday and Friday, at a specified time (for example, 10:00), which further reduces pharmacy costs and limits medication errors.

Pediatrics has led the way in infectious disease prevention and now we can alleviate one cause of nosocomial infection in preterm infants and prevention should be instituted in every NICU. With single center and multicenter randomized controlled studies and a meta-analysis demonstrating a $91 \%$ decrease in ICI in infants $<1000$ g, fluconazole prophylaxis should be targeted to this group of infants $<1000 \mathrm{~g}$ or $\leqslant 27$ weeks due to the high mortality and NDI. The prevention of ICI in extremely preterm infants also eliminates Candida as a cause of mortality and NDI in these vulnerable hosts.

DA Kaufman

Department of Pediatrics, University of Virginia Medical School,

Charlottesville, VA, USA

E-mail:dak4r@virginia.edu

\section{References}

1 Fridkin SK, Kaufman D, Edwards JR, Shetty S, Horan T. Changing incidence of Candida bloodstream infections among NICU patients in the United States: 1995-2004. Pediatrics 2006; 117: 1680-1687.

2 Cotten CM, McDonald S, Stoll B, Goldberg RN, Poole K, Benjamin Jr DK. The association of third-generation cephalosporin use and invasive candidiasis in extremely low birth-weight infants. Pediatrics 2006; 118: 717-722.

3 Benjamin Jr DK, Stoll BJ, Fanaroff AA, McDonald SA, Oh W, Higgins RD et al. Neonatal candidiasis among extremely low birth weight infants: risk factors, mortality rates, and neurodevelopmental outcomes at 18-22 months. Pediatrics 2006; 117: 84-92.

4 Stoll BJ, Hansen NI, Adams-Chapman I, Fanaroff AA, Hintz SR, Vohr B et al. Neurodevelopmental and growth impairment among extremely low-birth-weight infants with neonatal infection. JAMA 2004; 292: 2357-2365.

5 Kaufman D, Boyle R, Hazen KC, Patrie JT, Robinson M, Donowitz LG. Fluconazole prophylaxis against fungal colonization and infection in preterm infants. $N$ Engl J Med 2001; 345: 1660-1666.

6 Kicklighter SD, Springer SC, Cox T, Hulsey TC, Turner RB. Fluconazole for prophylaxis against candidal rectal colonization in the very low birth weight infant. Pediatrics 2001; 107: 293-298.

7 Kaufman D, Boyle R, Hazen KC, Patrie JT, Robinson M, Grossman LB. Twice weekly fluconazole prophylaxis for prevention of invasive Candida infection in high-risk infants of <1000 grams birth weight. J Pediatr 2005; 147: 172-179.

8 Manzoni P, Stolfi I, Pugni L, Decembrino L, Magnani C, Vetrano G et al. A multicenter, randomized trial of prophylactic fluconazole in preterm neonates. $\mathrm{N} \mathrm{Engl}$ J Med 2007; 356: 2483-2495.

9 Healy CM, Baker CJ, Zaccaria E, Campbell JR. Impact of fluconazole prophylaxis on incidence and outcome of invasive candidiasis in a neonatal intensive care unit. J Pediatr 2005; 147: 166-171.

10 Bertini G, Perugi S, Dani C, Filippi L, Pratesi S, Rubaltelli FF. Fluconazole prophylaxis prevents invasive fungal infection in high-risk, very low birth weight infants. J Pediatr 2005; 147: 162-165.

11 Manzoni P, Arisio R, Mostert M, Leonessa M, Farina D, Latino MA et al. Prophylactic fluconazole is effective in preventing fungal colonization and fungal systemic infections in preterm neonates: a single-center, 6-year, retrospective cohort study 2. Pediatrics 2006; 117: e22-e32.

12 Uko S, Soghier LM, Vega M, Marsh J, Reinersman GT, Herring L et al. Targeted shortterm fluconazole prophylaxis among very low birth weight and extremely low birth weight infants. Pediatrics 2006; 117: 1243-1252.

13 Aghai ZH, Mudduluru M, Nakhla TA, Amendolia B, Longo D, Kemble N et al. Fluconazole prophylaxis in extremely low birth weight infants: association with cholestasis. J Perinatol 2006; 26: 550-555.

14 Smith PB, Morgan J, Benjamin JD, Fridkin SK, Sanza LT, Harrison LH et al. Excess costs of hospital care associated with neonatal candidemia. Pediatr Infect Dis J 2007; 26: $197-200$.

15 Zaoutis TE, Heydon K, Localio R, Walsh TJ, Feudtner C. Outcomes attributable to neonatal candidiasis. Clin Infect Dis 2007; 44: 1187-1193.

16 Marr KA, Seidel K, White TC, Bowden RA. Candidemia in allogeneic blood and marrow transplant recipients: evolution of risk factors after the adoption of prophylactic fluconazole. J Infect Dis 2000; 181: 309-316. 
17 Manzoni P, Mostert M, Latino MA, Arisio R, Leonessa M, Farina D et al. Routinary use of fluconazole prophylaxis in a neonatal intensive care unit does not select natively fluconazole-resistant Candida subspecies. Pediatr Infect Dis J 2008 In press.

18 Sarvikivi E, Lyytikainen 0, Soll DR, Pujol C, Pfaller MA, Richardson M et al. Emergence of fluconazole resistance in a Candida parapsilosis strain that caused infections in a neonatal intensive care unit 3. J Clin Microbiol 2005; 43: 2729-2735.

19 Yoder BA, Sutton DA, Winter V, Coalson JJ. Resistant Candida parapsilosis associated with long term fluconazole prophylaxis in an animal model. Pediatr Infect Dis J 2004; 23: $687-688$.

20 Stoll BJ, Hansen N, Fanaroff AA, Wright LL, Carlo WA, Ehrenkranz RA et al. To tap or not to tap: high likelihood of meningitis without sepsis among very low birth weight infants. Pediatrics 2004; 113: 1181-1186.
21 Kaufman D, Fairchild KD. Clinical microbiology of bacterial and fungal sepsis in verylow-birth-weight infants. Clin Microbiol Rev 2004; 17: 638-680.

22 Johnsson $\mathrm{H}$, Ewald $\mathrm{U}$. The rate of candidaemia in preterm infants born at a gestational age of 23-28 weeks is inversely correlated to gestational age. Acta Paediatr 2004; 93 954-958.

23 Makhoul IR, Bental Y, Weisbrod M, Sujov P, Lusky A, Reichman B. Candidal versus bacterial late-onset sepsis in very low birthweight infants in Israel: a national survey. J Hosp Infect 2007; 65: 237-243.

24 Hack M. Neonatology fellowship training in research pertaining to development and follow-up 7. J Perinatol 2006; 26(Suppl 2): S30-S33.

25 Kramer MS, Platt RW, Wen SW, Joseph KS, Allen A, Abrahamowicz M et al. A new and improved population-based Canadian reference for birth weight for gestational age 6 . Pediatrics 2001; 108: E35. 